\title{
REVISED VERSION: \\ SIMULATION OF THE PRIMARY JET BREAKUP OF NON-NEWTONIAN FUELS: BASIC RESEARCH FOR SIMULATION-ASSISTED DESIGN OF LOW-GRADE FUEL BURNER
}

\author{
Thomas Müller* \\ Kathrin Kadel \\ Peter Habisreuther \\ Dimosthenis Trimis \\ Nikolaos Zarzalis \\ Engler-Bunter-Institute \\ Karlsruhe Institute of Technology (KIT) \\ Karlsruhe, Germany
}

\author{
Alexander Sänger \\ Tobias Jakobs \\ Thomas Kolb \\ Institute for Technical Chemistry \\ Karlsruhe Institute of Technology \\ Karlsruhe, Germany
}

\begin{abstract}
The research work of the present study is focused on the numerical simulation of primary breakup of high-viscosity nonNewtonian fluids. For the experimental investigation of fluid properties such as viscosity, surface tension and flow behaviour on the jet breakup an external mixing twin-fluid nozzle is used, as investigated in a previous study [Müller et al., ASME Turbo Expo 2016, GT2016-56371]. To describe the disintegration process of the fluids, characteristic features like liquid jet morphology, breakup length, breakup frequency and spray angle are evaluated. Furthermore, the primary breakup of slurries is simulated without discretizing the particles as a third phase, which heavily reduced the computational effort. Instead, the physical properties (density, viscosity) of the liquid phase take the influence of the particles into account.

The primary breakup was investigated using the open source CFD software OpenFOAM. To gather the morphology of the primary breakup and the flow field characteristics compressible large eddy simulations (LES) were performed and the movement of the gas-liquid interface was captured by means of the Volume of Fluid-Method (VOF).
\end{abstract}

The conducted simulations showed good agreement with ex- perimental results with respect to the characteristic features (e.g. breakup length) and the significant influence of viscosity and surface tension on the primary breakup. It is reasonably justified that the used OpenFOAM code and VOF is sufficient to simulate the primary breakup of particle laden liquids without discretizing particles as a third phase. Moreover, those findings contribute to a better understanding of the physics responsible of the breakup of high-viscosity liquid jets and as well to create an experimentally validated CFD based tool for future burner development and optimization.

\section{NOMENCLATURE}

\section{Latin letters}

\begin{tabular}{|c|c|c|c|}
\hline$A$ & Area & $C$ & Fitting parameter \\
\hline$D$ & Diameter & $\mathrm{F}_{\sigma}$ & $\begin{array}{l}\text { Volumetric surface } \\
\text { tension force }\end{array}$ \\
\hline$L$ & Length & $K$ & Fitting parameter \\
\hline$P$ & Scalar pressure & $S_{f}$ & $\begin{array}{l}\text { Outward-pointing vector } \\
\text { of the cell face }\end{array}$ \\
\hline
\end{tabular}




$\begin{array}{llll}\text { SMD } & \text { Sauter mean diameter } & T & \text { Temperature } \\ U & \text { Mean velocity } & \mathrm{U} & \text { Velocity vector } \\ a & \text { Fitting parameter } & b & \text { Fitting parameter } \\ c_{v} & \begin{array}{l}\text { Specific volumetric } \\ \text { heat capacity }\end{array} & e & \text { Total energy } \\ f & \text { Frequency } & \mathrm{g} & \begin{array}{l}\text { Gravitational } \\ \text { acceleration }\end{array} \\ & \text { Fitting parameter } & \dot{m} & \text { Mass flow } \\ m & \text { Fitting parameter } & \dot{q} & \text { Conductive heat flux } \\ s & \text { Web thickness } & t & \text { Time } \\ x, y, z & \text { Directions in space } & & \end{array}$

$x, y, z \quad$ Directions in space

\section{Greek letters}

$\begin{array}{llll}\alpha & \text { Volume fraction } & \dot{\gamma} & \text { Shear rate } \\ \kappa & \text { Curvature of the } & \mu & \text { Dynamic viscosity } \\ & \text { surface } & & \\ \phi & \text { Arbitrary variable } & \psi & \text { Compressibility } \\ \rho & \text { Density } & \sigma & \text { Surface tension } \\ \tau & \text { Viscous stress tensor } & & \end{array}$

\section{Non-dimensional parameters}

$\begin{array}{ll}R e & \text { Reynolds number } \\ \text { Oh } & \text { Ohnesorge number } \\ \text { GLR } & \text { Gas-liquid ratio }\end{array}$

$\begin{array}{ll}\text { We } & \text { Weber number } \\ M & \text { Momentum ratio }\end{array}$

\section{Subscripts}

$\begin{array}{llll}b & \text { Breakup } & c & \text { Compression } \\ f & \text { Based on the cell face } & g & \text { Gas } \\ h & \text { Hydraulic } & l & \text { Liquid } \\ \max & \text { Maximum } & \text { rel } & \text { Relative gas/liquid } \\ 0 & \text { Zero shear } & \infty & \text { Infinite shear }\end{array}$

\section{MOTIVATION AND INTRODUCTION}

Shortage of conventional fuels and the necessity to reduce $\mathrm{CO}_{2}$-emissions lead to an increased usage of low quality fuels such as pyrolysis oils and coal-water-slurries (CWS). In terms of atomisation, the most challenging aspect of such liquids is their complex atomisation behaviour due to the wide range of physical properties like viscosity and surface tension as well as the shear dependent flow behaviour.

One way to utilize such low-grade fossil or biogenic liquid fuels is their conversion to synthesis gas (main components: $\mathrm{CO}$ $\& \mathrm{H}_{2}$ ), e.g. via high pressure entrained flow gasification (EFG). The EFG has a wide variety of applications [1]. Thus, the syngas can be used for the synthesis of methane (SNG), for the production of high quality speciality chemicals via a synthesis $(\mathrm{CtL}$; BtL) or serve as fuel in an IGCC power plant.

For this application field mainly gas assisted burner nozzles are used due to complex physical properties of the fuels, as they allow low liquid velocities and therefore resonable pres- sure drops inspite of the high-viscosities. As fuel conversion is driven by droplet evaporation and subsequent degradation of the evaporated fuel, the spray quality, the spray angle and flow field generated by the burner nozzle are of major importance [2] to produce a high quality syngas.

Due to the specific operating conditions the atomisation for EFG is connected with several challenges, (I) high viscosity (up to $1000 \mathrm{mPas}$ ), (II) complex rheological behaviour, (III) operation at elevated reactor pressure (up to $80 \mathrm{bar}$ ), (IV) operation in oxygen-blown mode, where the atomisation agent also serves as gasification agent to increase the overall efficiency of the process. As the amount of gasification agent is limited by the stoichiometry of the reaction, the available amount of atomisation agent is limited, too. Since the spray quality decreases with the amount of available atomisation agent, a thorough understanding of the atomisation process of high-viscosity fuels is needed.

The liquid jet breakup in external mixing twin-fluid nozzles is characterized by the following non-dimensional parameters: The liquid (Eqn. (1) and gas Reynolds numbers (Eqn. (4), the aerodynamic Weber number (Eqn. (2)), the Ohnesorge number Eqn. (5), the momentum flux ratio (Eqn. (3) and the gas-liquid ratio (Eqn. (6)

$$
\begin{array}{lll}
R e_{l}=\frac{\rho_{l} U_{l} D_{l}}{\mu_{l}} & \text { (1) } & R e_{g}=\frac{\rho_{g} U_{g} D_{h}}{\mu_{g}} \\
W e=\frac{\rho_{g} U_{r e l}^{2} D_{l}}{\sigma} & \text { (2) } & O h=\frac{\mu_{l}}{\sqrt{\rho_{l} \sigma D_{l}}} \\
M=\frac{\rho_{g} U_{g}^{2}}{\rho_{l} U_{l}^{2}} & \text { (3) } & G L R=\frac{\dot{m}_{g}}{\dot{m}_{l}}
\end{array}
$$

Here, $U_{g}$ and $U_{l}$ are the mean gas and liquid velocitiy and $U_{r e l}$ is the relative gas/liquid velocity, whereas $D_{h}$, in Eqn. (4), is the hydraulic diameter and is defined as twice the annular gap width of the gas channel.

The application of twin-fluid nozzles for the atomisation of low viscosity liquids at ambient pressure has been thoroughly studied in literature and an overview of the experimental investigations is provided by [3]: For example, [4-8] investigated among other things: the breakup regimes, the liquid core lengths and the breakup frequencies of coaxial air-water jets. In addition, the liquid core length was also studied by [9-11], whereas the influence of web thickness was studied for instance by [12]. The breakup of a water jet at reactor pressures up to 21 bar was studied by [13]. The breakup morphology, breakup length and oscillation frequency of coke water slurries in an annular air jet were determined by Zhao et al. [14] . Tsai and $\mathrm{Vu}[15]$ showed the impact of slurry viscosity on airblast atomisation quality with an internal mixing twin-fluid jet atomizer but also the influence 
of the particle size distribution.

A correlation to predict the Sauter mean diameter (SMD) based on non-dimensional parameters is provided by [16] and shown in Eqn. (7),

$S M D=C D_{l} O h^{m}(G L R W e)^{n}$,

where $C, m, n$ are fitting parameters. For a better understanding of the complex rheological behaviour and its effect upon the atomisation a method to estimate the apparent viscosity under atomisation conditions was deduced in [17]. Sänger et al. showed [17], that this correlation (Eqn. (7)) can also be used to predict droplet sizes of non-Newtonian liquids, if the correct value of the shear dependent viscosity during the atomisation process is used in the Ohnesorg-Number, which emphasizes the need to correctly predict this property.

The experimental investigations of laminar liquid jets showed, that the breakup process can be seperated into two stages: the destabilization due to the high velocity difference between the surrounding gas stream and the liquid jet, which induces disturbances on the jet surface due to a Kelvin-Helmholtz like instability [8]. As these pertubations grow, the jet is undergoing an increasing deformation. This second stage begins, as liquid structures are deflected into the gas stream and finally disintegrate to ligaments and large droplets.

Contrary to the experimental investigations, numerical studies focusing on the primary jet breakup in gas assisted nozzles are still scarce and also mostly limited to low viscosity liquids. For instance, Tian et al. [18] studied the primary breakup of a water jet. An overview upon the modelling of primary atomisation can be found in [19].

Analysis of high-speed images from a previous study by the authors showed [20], that the breakup morphology changes from the membrane (Fig. 1a, left) to the fiber type breakup (Fig. 1a, right) with decreasing liquid viscosity or increasing $G L R$.

Furthermore it was determined, that the jet disintegration follows two different types of instability depending on liquid viscosity and $G L R$, as illustrated in Fig. 1b: A pulsating mode consisting of a longitudinal and axis-symmetric mode triggered by a Kelvin-Helmholtz instability (Fig. 1b, left) and a flapping mode characterized by a deflection in radial direction shortly after the nozzle (Fig. 1b, right). Moreover, a transition between both modes was detected for some operating conditions. Additionally, droplet size measurements showed [20], that the flapping instability mode results in a wider spray angle and lower SMD than the pulsating mode.

In addition, the influence of reactor pressure upon the atomisation of high-viscosity liquids was studied in [21], where a decrease of drop size for a constant relative velocity between gas and liquid was reported with increasing reactor pressure. Moreover the stabilization of suspension fuels as well as its impact

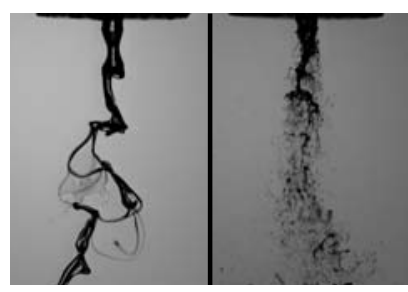

(a) BREAKUP REGIMES: MEMBRANE (LEFT) AND FIBER TYPE (RIGHT)[20]

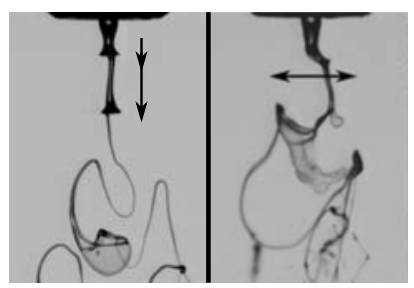

(b) INSTABLITY MODES: PULSATING (LEFT) AND FLAPPING (RIGHT)[20]

\section{FIGURE 1: MORPHOLOGY OF LIQUID JET BREAKUP}

upon the atomisation performance was studied in [22]. They showed, that by the creation of a capillary suspension the stability of the fuel was increased as well as the spray quality was improved. In this context the dependency of spray angles on surface tension was evaluated. In addition, a change of the primary instability mode from flapping to pulsating was reported, when the secondary fluid was added. Moreover, gasification experiments were conducted with an atmospheric lab-scale plant, which used an external mixing twin-fluid nozzle similar to the ones investigated here [2].

Besides these experiments, numerical simulations were conducted by the authors to investigate the primary breakup of highviscosity liquids in [23-25] and validated with the data obtained in the experiments. These results showed good agreement with the measurements with regard to the breakup morphology and the mode of the primary instability as well as the breakup frequency and length.

The objective of this study is the investigation of the influence of surface tension and non-Newtonian behaviour upon the primary breakup process by numerical simulation. Secondly, the simulation of a coke water slurry is considered by using a simplified approach, which reduces the computational effort.

\section{NUMERICAL AND RHEOLOGIC FORMULATION}

The two-phase simulations in this study were performed using the compressibleInterFoam-code of the open source $\mathrm{C}++$ libraries of OpenFOAM [26] (Version 2.4.0), which applies the Volume of Fluid-Method (VOF) by Hirt and Nichols [27] to capture the interface. The upcoming section will give a short overview upon the modelling of the liquid jet breakup, especially with regard to the behaviour and modelling of non-Newtonian fluids. For a more detailed description the reader is referred to previous publications [23,24].

\section{Governing equations}

For the numerical description of the underlying two-phase problem the governing equations for mass (Eqn. (8)), momentum 
(Eqn. (9) ) and energy (Eqn. (10) have to be solved as well as an equation, that describes the interface between the two phases based on the continuity equation of the liquid (Eqn. (11)):

$$
\begin{aligned}
& \frac{\partial \rho}{\partial t}+\nabla \cdot(\rho \mathrm{U})=0 \\
& \frac{\partial(\rho \mathrm{U})}{\partial t}+\nabla \cdot(\rho \mathrm{UU})=-\nabla P+\nabla \cdot \tau+\rho \mathrm{g}+\mathrm{F}_{\sigma} \\
& \frac{\partial(\rho e)}{\partial t}+\nabla \cdot(\rho e \mathrm{U})=-\nabla \cdot(P \mathrm{U})+\nabla \cdot(\tau \mathrm{U})-\nabla \dot{q} \\
& \frac{\partial\left(\rho_{l} \alpha_{l}\right)}{\partial t}+\nabla \cdot\left(\rho_{l} \alpha_{l} \mathrm{U}_{l}\right)=0
\end{aligned}
$$

$\tau$ represents the viscous stress tensor and is defined as $\tau=$ $2 \mu\left(0.5\left[(\nabla \mathrm{U})+\left(\nabla \mathrm{U}^{\top}\right)\right]\right)$, while $e$ is total energy with $e=c_{v} T+$ $0.5|\mathrm{U}|^{2}$. It should be noted, that only properties labelled with a subscript $l$ for the liquid phase correspond to the properties of the respective liquid, whereas all other properties without a subscript correspond to the single continuous fluid according to the VOF method, which will be discussed in the next sub-section.

\section{Interface capturing}

The VOF method is based on the representation of the interface by a single variable for each computational cell, which is transported by advection with the flow field. In addition, gas and liquid phase are considered one hypothetical continuous fluid throughout the domain. The volume fraction of the liquid is commonly used as indicator function of the interface and as such defined in the range of $\alpha_{l} \in[0,1]$, where the liquid phase is represented by $\alpha_{l}=1$ and the gas phase by $\alpha_{l}=0$. For the sake of better readability the liquid volume fraction is from here on denoted without subscript $\alpha_{l} \equiv \alpha$. Combination of Eqn. (11) and (8) and subsequent re-formulation leads to the interface capturing advection equation of the liquid volume fraction $\alpha$ :

$$
\begin{aligned}
\frac{\partial \alpha}{\partial t}+\nabla \cdot(\mathrm{U} \alpha)+\nabla & \cdot\left(\alpha(1-\alpha) \mathrm{U}_{c}\right) \\
& =\alpha(1-\alpha)\left(\frac{\psi_{g}}{\rho_{g}}-\frac{\psi_{l}}{\rho_{l}}\right) \frac{D P}{D t}+\alpha \nabla \cdot \mathrm{U}
\end{aligned}
$$

The properties of the hypothetical continuous fluid such as density $\rho$ or viscosity $\mu$ are calculated for an arbitrary volumetric property $\phi$ by an average of the properties of the gas $\phi_{g}$ and liquid phase $\phi_{l}$, weighted with the liquid volume fraction $\alpha$ according to [28]:

$\phi=\phi_{l} \alpha+\phi_{g}(1-\alpha)$

The volumetric surface tension force in Eqn. (9) is calculated with the CSF model proposed by Brackbill et al. [29]:

$\mathrm{F}_{\sigma}=\sigma \kappa(\alpha) \nabla \alpha, \quad$ with $\quad \kappa(\alpha)=-\nabla \cdot\left(\frac{\nabla \alpha_{f}}{\left|\nabla \alpha_{f}\right|} \cdot S_{f}\right)$

The correct calculation of the surface tension force is highly dependent upon a sharp resolution of the interface, which is represented by all cells with $\alpha \in] 0,1[$ and typically extends over several cells. This effect leads to a smearing of the precise interface, which is commonly known as numerical diffusion, and is influenced by grid resolution as well as the discretization scheme of the first divergence term in Eqn. (12).

In order to minimize the "smearing" an artificial interface compression velocity $\mathrm{U}_{c}$ is used in the second divergence term in Eqn. (12) following the algebraic counter-gradient approach by Weller [30]. The compression velocity is calculated as formulated in [28], which limits the compression to the direction normal to the surface and prevents unwanted advection of the liquid volume fraction in other directions. Due to the factor $\alpha(1-\alpha)$ the compression is also limited to the area of gas-liquid surface, as it is only there differing from zero.

To guarantee boundedness of the liquid volume fraction Eqn. (12) is solved using the MULES (Multidimensional Universal Limiter for Explicit Solutions) method [31] in OpenFOAM, which uses an additional limiter to cut-off of the face-fluxes during advection.

\section{Turbulence modelling}

As the proper prediction of the transient turbulent gas flow is decisive for the modelling of the atomisation process in twinfluid nozzles, an LES approach is chosen, based on spatial filtering of the flow field [32]. An algebraic Smagorinsky model [33 was used for the calculation of the unknown subgrid-scale stress tensor, which is further detailed in the work of Fureby [34].

A detailed reasoning for the choice of the subgrid-scale model as well as a comparison of the calculated flow field with experimental measurements is given in a previous publication [23]. 


\section{Shear thinning behaviour}

Shear thinning non-Newtonian fluids show a different flow behaviour than Newtonian ones. Their viscosity decreases with the strength of the shear force and has to be calculated during the simulation. The shear thinning behaviour as function of the shear rate can be estimated by a model equation. Possible equations are the Bird-Carreau-model (Eqn. (15)) proposed by Bird and Carreau [35, 36], the Cross-model (Eqn. (16)) by Cross [37] or the Sisko-model (Eqn. (17)), derived from Sisko [38]. The models contain the shear rate $\dot{\gamma}$, the zero viscosity $\mu_{l, 0}=\mu_{l}(\dot{\gamma} \rightarrow 0)$, the infinite viscosity $\mu_{l, \infty}=\mu_{l}(\dot{\gamma} \rightarrow \infty)$ and various fitting parameters $K, n, a$ and $b$.

$\mu_{l}=\frac{\mu_{l, 0}-\mu_{l, \infty}}{\left(1+(K \dot{\gamma})^{2}\right)^{\frac{1-n}{2}}}$

$\mu_{l}=\frac{\mu_{l, 0}-\mu_{l, \infty}}{1+(K \dot{\gamma})^{n}}$

$\mu_{l}=a+b \dot{\gamma}^{n-1}$

The choice of model equation depends on the respective fluid. These three model equations have displayed the best agreements with experimental results based upon analysis of sheardependent viscosity using a rotational rheometer (Searle-type).

\section{SETUP}

This section presents the preceding study conducted in order to validate the implementation of the model equations (Eqn. (15)-(17)). In addition, the experimental test rig, the investigated twin-fluid nozzle and the properties of the used model liquids and the atomisation agent are introduced. Moreover, the numerical domain and the boundary conditions are illustrated.

\section{Preliminary study: Two-plates model}

The schematic and the numerical domain of the Two-plates model, which was used in a preceding study to validate the model equations (Eqn. (15) - (17)), is shown in Fig. 2. More details about the model equations are presented in [39]. A Couette flow is adjusted by the flow velocity and results in a constant shear rate $\dot{\gamma}=\frac{d U}{d y}=$ const. Therefore the velocity of the upper plate is set to a maximal velocity $U_{\max }$ and the velocity of the lower plate is set to zero.
The boundary condition for the velocity field at the inlet and outlet is given by a zero-gradient approach. The pressure boundary conditions on the upper and lower plate is configured so that the flow is determined by the velocity boundary condition. On the inlet the pressure is set to a constant value of 1 bar and at the outlet a zero-gradient condition is used. The liquid volume fraction and temperature are set to values of 1 and $293 \mathrm{~K}$, respectively, at the inlet and in the computational domain, whereas a zero-gradient approach is used for all other boundaries.

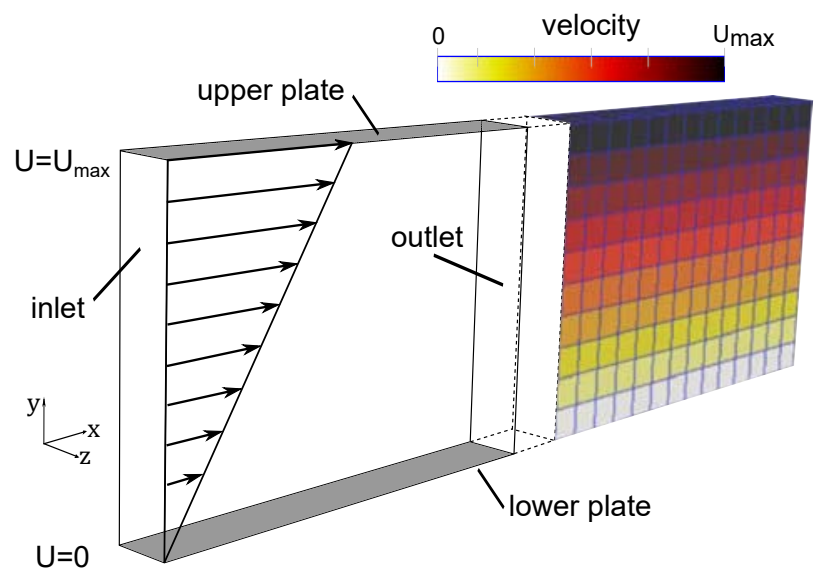

FIGURE 2: SCHEMATIC AND NUMERICAL DOMAIN OF THE TWO-PLATES MODEL WITH VELOCITY PROFILE

\section{Experimentally investigated atomizer nozzle}

The measurements presented in this work were conducted at the ATMO test rig. A detailed description of it is given in [20].

Experiments were performed using an external mixing twin fluid nozzle, which is schematically presented in Fig. 3. The liquid is supplied by a central tube to the nozzle orifice whereas atomisation agent flows through a concentric annular gap. The central liquid tube is coloured blue in Fig. 3 and has a diameter of $D_{l}=2 \mathrm{~mm}$, while the diameter of the outer annular gas gap at the exit plane of the nozzle is $D_{g}=5.4 \mathrm{~mm}$ and is coloured in green.

\section{Numerical domain}

The numerical domain of the nozzle geometry is depicted in Fig. 4 as half-section of the entire domain, cropped in $\mathrm{x}$-direction approximately $50 \mathrm{~mm}$ after the nozzle exit area for the sake of better visualization. The region of the primary breakup as well as the internal of the twin-fluid nozzles are covered by the computational grid. The internal of the nozzles was included as it 


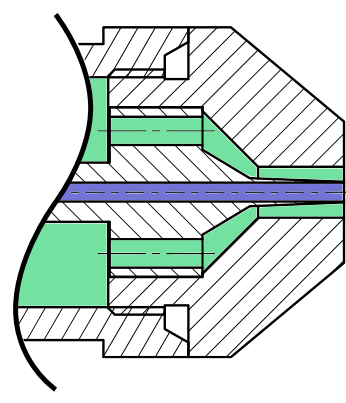

(a) SIDE VIEW

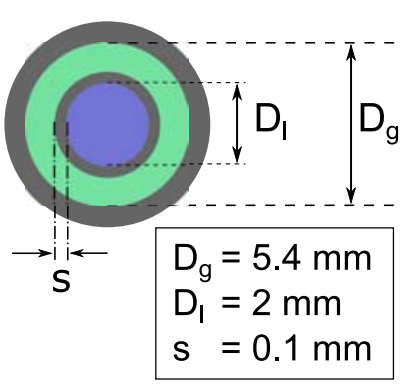

(b) FRONT VIEW
FIGURE 3: SCHEMATIC OF THE INVESTIGATED TWIN FLUID NOZZLE

might influence the gas velocity field, which in turn influences the atomisation. A cylinder with a radius of $0.015 \mathrm{~m}$ and a length of $0.1 \mathrm{~m}$ is used to discretise the external flow domain.

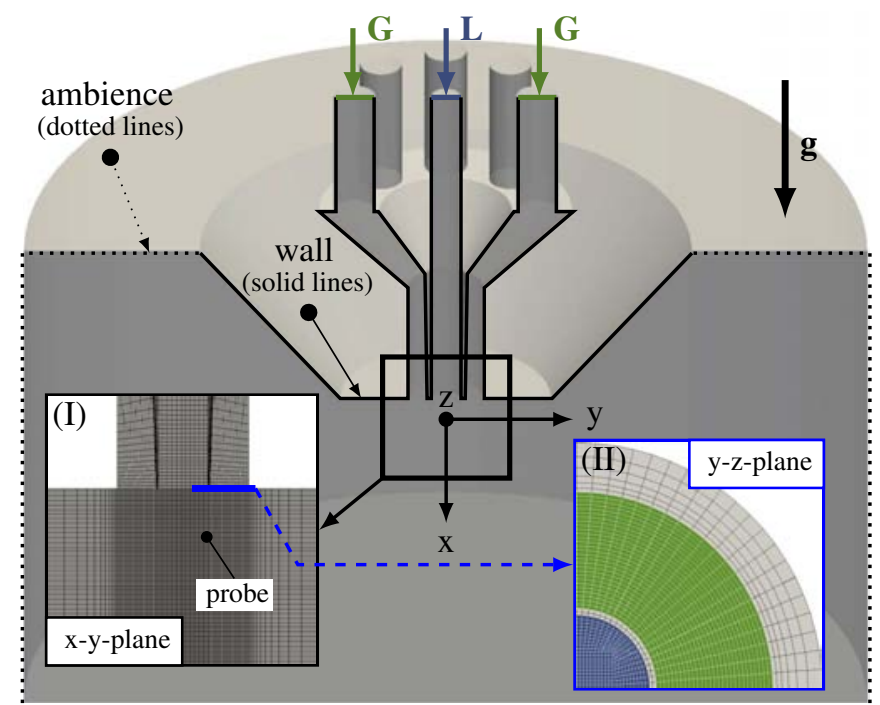

FIGURE 4: NUMERICAL DOMAIN OF THE TWO-PHASE SIMULATIONS

The grid is composed of hexahedral cells, which start from a central o-grid in the y-z-plane, see Fig. 4, detail (II). The o-grid's central block has a resolution of $50 \mu \mathrm{m}$ in all three directions. A cut of the nozzle exit area in the $x-y-p l a n e$ of the mesh is shown in Fig. 4, detail (I).

\section{Boundary conditions and fluid properties}

A non-slip boundary condition was set for the velocity at the walls, whereas the inlet profiles of gas and liquid were initialized with a uniform velocity corresponding to the given mass flows. At the ambience a zero-gradient condition was used for the velocity field and the pressure was set to $1 \mathrm{bar}_{\mathrm{abs}}$. The temperature was set to $20^{\circ} \mathrm{C}$ at all inlets and a zero-gradient approach was used otherwise. The liquid phase fraction value was set to 1 at the liquid inlet and to 0 at the gas inlet, respectively.

To investigate the influence of the surface tension and the dynamic shear viscosity on primary jet breakup, different nonNewtonian and highly viscous Newtonian fluids were investigated: A china clay/glycerol/water suspension (china clay mass fraction: $15 \%$, glycerol mass fraction: $71.8 \%$, china clay particle size: approx. $2 \mu \mathrm{m}$, acronym : $C C G[17])$, a carboxymethylcellulose/water mixture (acronym: $C M C$ ) and a beech wood coke/water suspension with and without addition of $3.1 \%$ octanol (coke particle volumetric mean equivalent sphere diameter: $20 \mu \mathrm{m}$, acronym: $C W S$ ) were analysed as non-Newtonian fluids. To compare the behaviour of the non-Newtonian with the Newtonian fluids a glycerol/water mixture with the constant viscosity of $400 \mathrm{mPas}$ (glycerol mass fraction: $93.8 \%$, [17],23], acronym: $G L W$ ) was used.

With a rheometer different viscosities are determinated by varying shear rate. Hence, zero and infinite viscosity of each model fluid can be calculated by extrapolation using Eqn. (15) - (17). Both viscosities and the fitting parameters of the model fluid are listed in Tab. 1 with the appropriate model equation. The dynamic viscosity was measured within shear rates of $\dot{\gamma}=1-$ $4000 \mathrm{~s}^{-1}$ using a rheometer with a cylindrical measuring system (Searle-type), see Searle [40]. The very good agreement between model equations and the measurement data is also depicted in Fig. 5 .

Surface tension $\sigma$ and liquid density $\rho_{l}$ were measured with a tensiometer applying the Du Noüy ring method, see du Noüy [41] and the weighing method for liquid density.

All other physical properties of the investigated model liquids, which were measured at $20^{\circ} \mathrm{C}$ and $1 \mathrm{bar}$, as well as the operating conditions and the acronyms for each simulation are given in Tab. 2 Air was used as atomisation agent with a density of $1.205 \mathrm{~kg} \mathrm{~m}^{-3}$ and a dynamic viscosity of $0.0182 \mathrm{mPas}$.

\section{RESULTS}

An overview about the physical properties of the model fluids and the operating conditions of the simulations is given in Tab. 2 The liquid mass flow was set to a constant value of $10 \mathrm{~kg} \mathrm{~h}^{-1}$ during all simulations, while the varying gas mass flow implicates a $G L R$ between 0.5 and 0.8 .

Each of the simulation represents a physical time span of at least $50 \mathrm{~ms}$, which ensures an acceptable statistic convergence as at least 50 convective times are captured. The time stepping during a simulation is limited by the $C F L$-number to be smaller than 0.4 and results in time steps of approximately $0.25 \mu \mathrm{s}$. The simulated time span is then evaluated at $0.1 \mathrm{~ms}$, which is 400 
TABLE 1: PARAMETERS OF THE MODEL EQUATIONS

\begin{tabular}{|c|c|c|c|c|c|c|}
\hline Model fluid & Model equation & $\begin{array}{c}\mu_{0} \\
\mathrm{mPas}\end{array}$ & $\begin{array}{c}\mu_{\infty} \\
\mathrm{mPas}\end{array}$ & a & $\mathrm{b}$ & $\mathrm{n}$ \\
\hline Carboxymethylcellulose/water & Cross & 420 & 18 & \multicolumn{2}{|c|}{0.00602} & 0.62973 \\
\hline Beech wood coke/water ( $3.1 \%$ octanol) & Sisko & 199167 & 3.09 & 0.00309 & 0.714 & 0.045 \\
\hline
\end{tabular}

TABLE 2: PHYSICAL PROPERTIES OF THE MODEL FLUIDS AND OPERATING CONDITIONS AT $20^{\circ} \mathrm{C}$

\begin{tabular}{|c|c|c|c|c|c|c|}
\hline Case & $\begin{array}{c}\rho \\
\mathrm{kg} \mathrm{m}^{-3}\end{array}$ & $\begin{array}{c}\sigma \\
\mathrm{mNm}^{-1}\end{array}$ & $\begin{array}{c}\mu \\
\mathrm{mPas}\end{array}$ & $\begin{array}{c}G L R \\
-\end{array}$ & $\begin{array}{l}M \\
-\end{array}$ & $\begin{array}{c}\text { We } \\
-\end{array}$ \\
\hline$G L W 1$ & \multirow{3}{*}{1245} & 10 & \multirow{3}{*}{400} & \multirow{3}{*}{0.6} & \multirow{3}{*}{10.1} & 1239 \\
\hline$G L W 2$ [23] & & 64.1 & & & & 193 \\
\hline$G L W 3$ & & 100 & & & & 124 \\
\hline$C C G 1$ & \multirow{2}{*}{1325} & \multirow{2}{*}{64.9} & \multirow[t]{2}{*}{$*$} & 0.6 & 10.7 & 191 \\
\hline$C C G 2$ & & & & 0.8 & 19.0 & 341 \\
\hline$C M C$ & 1006 & 68.9 & $*$ & 0.6 & 8.1 & 179 \\
\hline$B W C l$ & 1099 & 71.7 & \multirow[t]{2}{*}{ * } & \multirow{2}{*}{0.5} & 6.2 & 119 \\
\hline$B W C 2$ & 1091 & 35.4 & & & 6.1 & 241 \\
\hline
\end{tabular}

GLW glycerol/water - glycerol mass fraction: $93.8 \%\left[\begin{array}{l|l|l|}17 & 23]\end{array}\right.$

CCG china clay/glycerol/water - china clay mass fraction: $15 \%$, glycerol mass fraction: $71.8 \%$ [17

CMC carboxymethylcellulose/water - carboxymethylcellulose mass fraction: $2.33 \%$ 17]

BWC beech wood coke/water - beech wood coke mass fraction: $20 \%$, BWC1: octanol volume fraction $0 \%$, BWC2: octanol volume fraction $3.1 \%$ [22]

* non-Newtonian fluid, dynamic viscosity is shear-dependent, see Tab. 1

times bigger than the computational time step of the simulation and makes frequency analysis possible in the range of $20 \mathrm{~Hz}$ to $5000 \mathrm{~Hz}$.

\section{Validation of the simulations}

Simulations of Newtonian fluids have already been validated with experiments with regard to morphology of the primary breakup and quantitative analysis of breakup frequency and length in previous investigations [23, 24].

To validate the correct calculation of the apparent viscosity during the simulation by the implemented model equations the previously described Two-plate model case is used. For this purpose, various shear rates between $0.1-1000 \mathrm{~s}^{-1}$ are adapted. The simulation then calculates the shear rate from the velocity field and subsequently the viscosity with the specified model equa- tion and parameters (Eqn. (15) - (17)). The value of the viscosity obtained from these simulations are then compared with the measured data and the consequent fit. As depicted in Fig. 5 the measurements and simulations show excellent agreement, thereof demonstrating the correct implementation of the model equations.

The simulations of non-Newtonian fluids are validated by comparison with experimental data with regard to breakup morphology, breakup length and frequency as well as the predicted apparent viscosity of the fluid. This is exemplarily shown for case $C M C$. Detailed information on the definitions of the breakup length and the breakup frequency as well as their determination can be found in [23].

Figure 6 depicts a snapshot of the liquid jet structure from experiment and simulation. For the simulations, projections of 


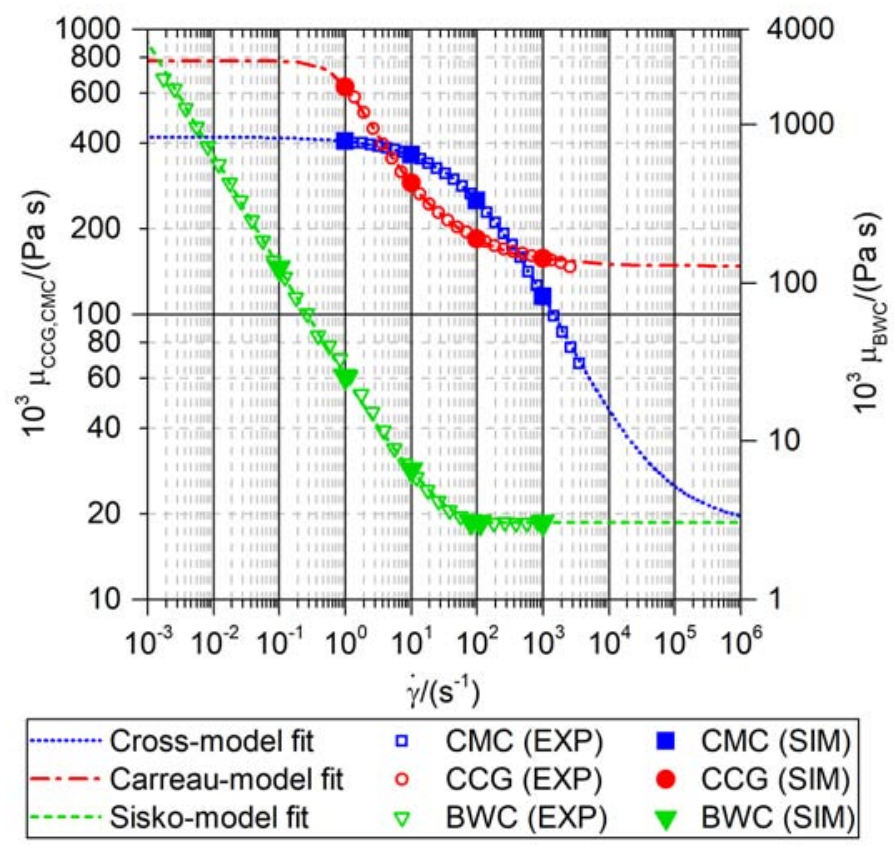

FIGURE 5: APPARENT VISCOSITY $\mu$ VS. SHEAR RATE $\dot{\gamma}$ : COMPARISON OF RHEOMETER MEASUREMENTS, MODEL EQUATIONS AND SIMULATIONS OF THE TWO-PLATES CASE

the liquid volume structure to the xy-plane (middle) as well as the xz-plane (right) are shown, as the jet breakup is a three dimensional phenomena. Comparison shows, that the breakup morphology is well captured by the simulation. The intact liquid structure shows the same features and characteristic length scales (emphasized by a vertical line). Furthermore, the simulation correctly predicts the formation of the axis-symmetric wave structure of the pulsation mode (marked by horizontal arrows), although with a slightly lower amplitude of the wave. In the experiment, the jet breakup follows the membrane type regime with the characteristic formation and disintegration of thin membranes. The formation of these membranes is well captured by the simulation, although the grid resolution of $50 \mu \mathrm{m}$ is not sufficient to reproduce the membrane itself, as their thickness is well below $5 \mu \mathrm{m}$. For a more detailed explanation of the limitations of the mesh resolution with regard to the membrane itself, the reader is referred to [23.

In Fig. 7 the temporal evolution of the breakup length $L_{b}$ is shown for the simulation of case $C M C$. Three different sections can be detected, which are marked with $I, I I$ and $I I I$, respectively. In section $I$ and $I I I$, the breakup length displays a periodic behaviour and approximately the same mean breakup length of $11 \mathrm{~mm}$, which is in good agreement with the experimental result of $10.8 \mathrm{~mm}$.

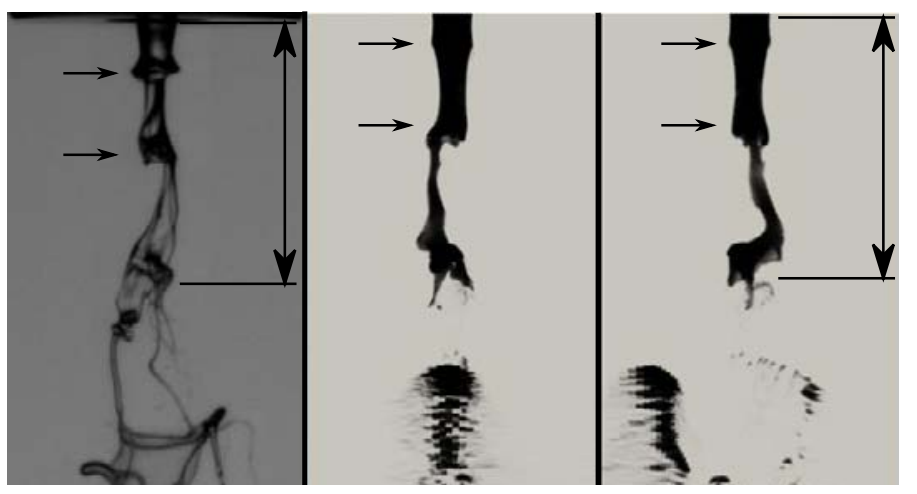

FIGURE 6: BREAKUP MORPHOLOGY OF CASE CMC. LEFT TO RIGHT: EXPERIMENT, SIMULATION (XYPLANE), SIMULATION (XZ-PLANE)

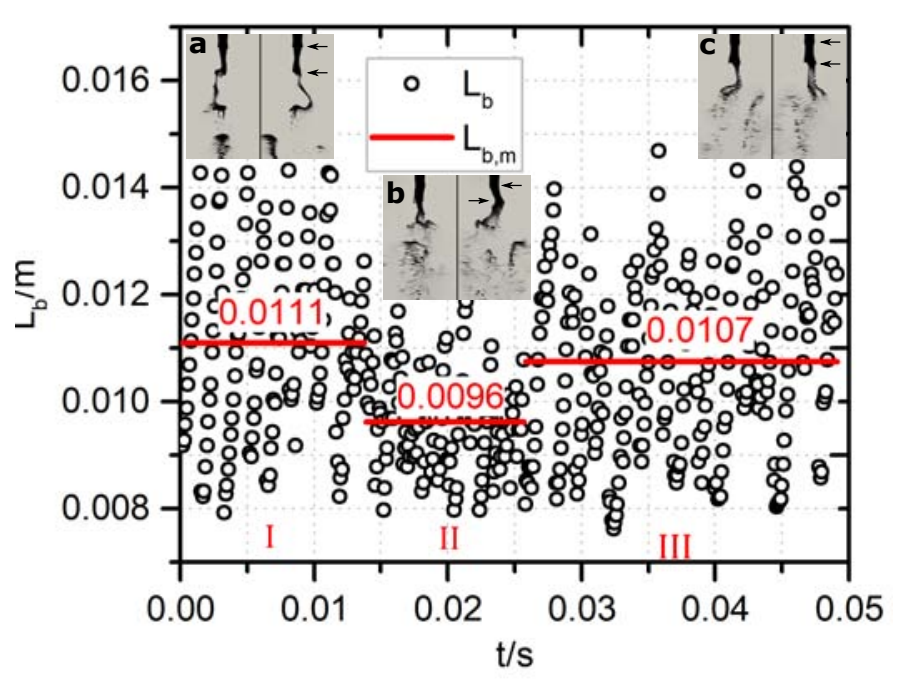

FIGURE 7: BREAKUP LENGTH $L_{b}$ VS. TIME $t$ (CASE $C M C$ )

In the transition region II the primary breakup mode changes to a slight flapping motion, which can be seen in the right part of picture (b) in Fig. 7 (emphasized by arrows), whereas region I and III follow the experiments and show a pulsating primary instability. The transition leads to a decrease of the breakup length to $9.6 \mathrm{~mm}$ and the periodic behaviour is not recognisable anymore.

This finding also is in accordance with previous investigations [25], wherein a change of the primary instability mode was induced by a slight change of the nozzle geometry at constant operating conditions and the same decrease in the liquid breakup length was reported.

Furthermore, the temporal behaviour of the jet breakup is studied in terms of the breakup frequency $f_{b}$, which is defined as the passing frequency of the axisymetric wave in case of the pul- 

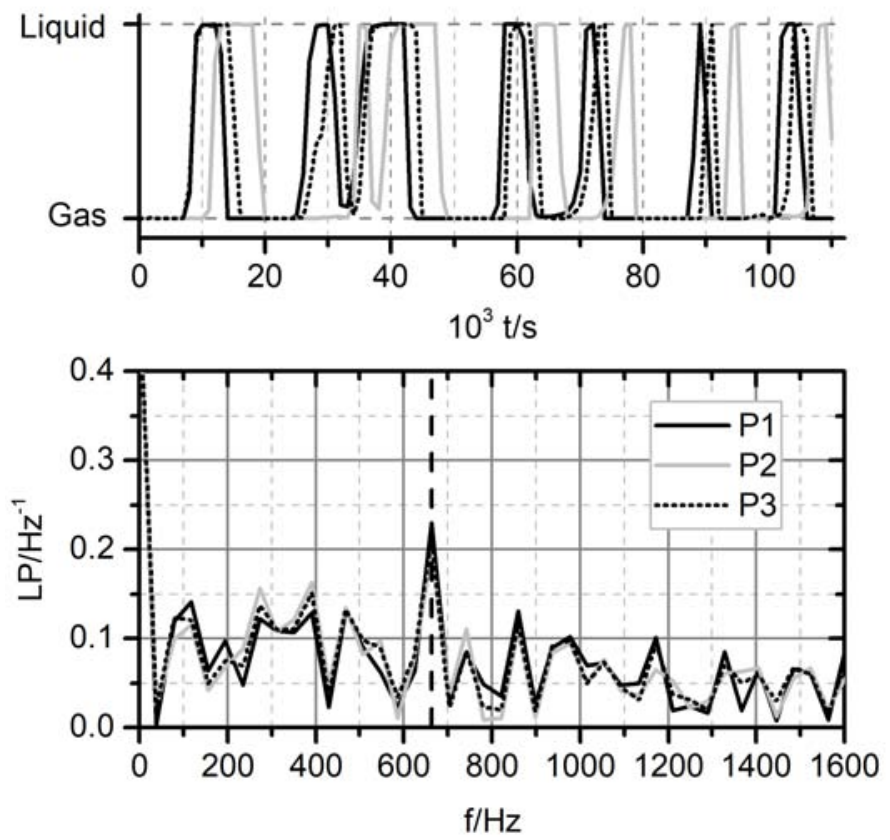

FIGURE 8: DETERMINATION OF THE BREAKUP FREQUENCY: LIQUID PRESENCE VS. TIME $t$ AT THREE VIRTUAL PROBES (TOP) AND LIQUID PRESENCE SPECTRUM $L P$ VS. FREQUENCY $f$ OF CASE $C M C$. THE VERTICAL LINE MARKS THE FUNDAMENTAL MODE

sating mode. Figure 8 exemplarily depicts the monitored liquid presence signal of three virtual probes (top) from region $I$ and the energy spectrum from a Fast Fourier transformation of these signals. The analysis yields a frequency of $664 \mathrm{~Hz}$, which is slightly lower than the measured value of $768 \mathrm{~Hz}$. Additionally, there is a good agreement between this frequency with the fundamental mode of the liquid breakup length signal of region $I$ and $I I I$ (see Fig. 7), which were determined to $640 \mathrm{~Hz}$ and $600 \mathrm{~Hz}$, respectively.

On top, the simulation was analysed with regard to the rheological behaviour and the effective viscosity is compared with the one experimentally determined. Sänger et al. [17] estimated the apparent viscosity of case $C M C$ during the jet breakup to $152 \mathrm{mPa}$. In Fig. 9 a cut of the liquid structure in the xy-plane is shown depicting the gas flow field in terms of vectors and the shear rate (left) as well as the effective viscosity (right) as contour plots. The shear rate is particularly large at liquid structures, that protrude into the gas stream, and as a consequence viscosity is low at these points. In contrast to this, the viscosity is lower at the center of the axis-symmetric instability. To determine the shear dependent viscosity in the simulation the liquid jet is cut in axial direction in blocks of $1 \mathrm{~mm}$ length, starting $1 \mathrm{~mm}$ upstream of the nozzle exit. The effective viscosity is then averaged over

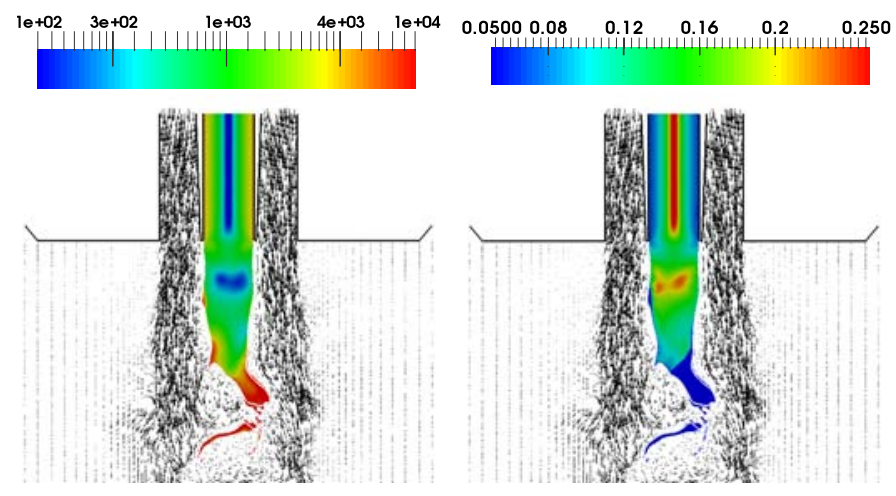

FIGURE 9: XY-CUTPLANE CLOSE TO THE NOZZLE EXIT: LEFT: SHEAR RATE. RIGHT: VISCOSITY. VECTORS ILLUSTRATE GAS FLOW FIELD.

all liquid cells in each of these blocks.

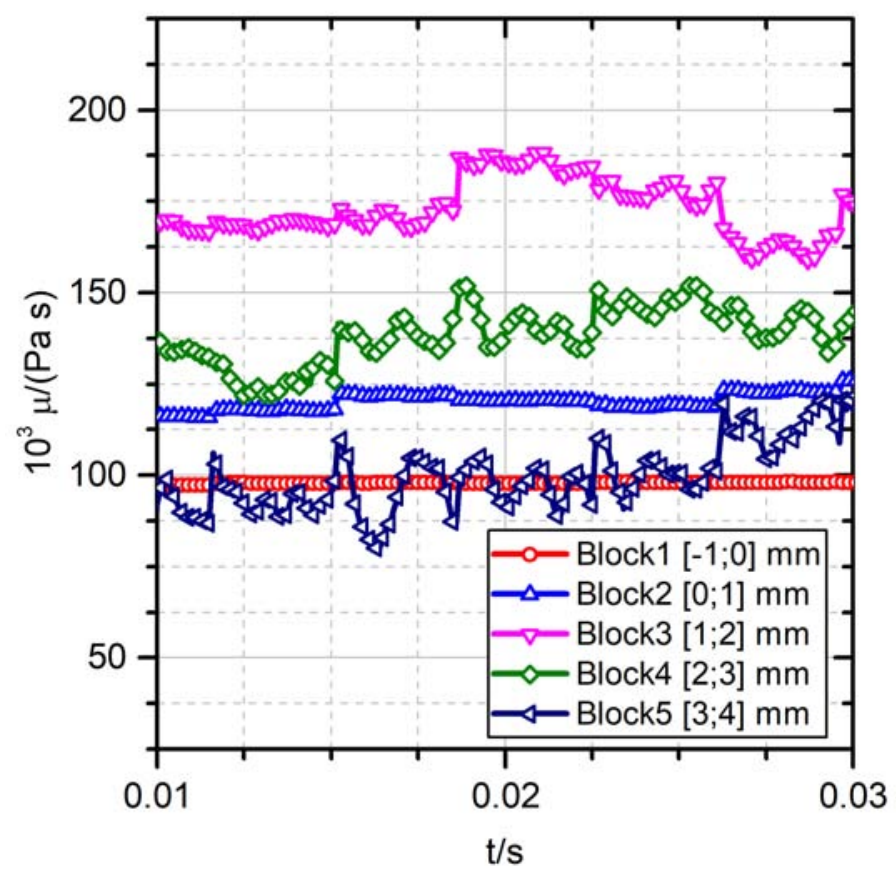

FIGURE 10: APPARENT VISCOSITY $\mu$ VS. TIME $t$

Figure 10 shows the temporal evolution of the apparent viscosity calculated for each of these blocks. For these blocks the mean apparent viscosity was calculated to values between $114.9 \mathrm{mPa}$ (Block5) and $169.1 \mathrm{mPa}$ (Block3). In addition, the average over blocks 3 to 4 was computed to $156.5 \mathrm{mPas}$, which is in good agreement with the experimentally determined value of $152 \mathrm{mPas}$. 
Deducing from the comparison of case $C M C$ with experiment the following simulations of other non-Newtonian fluids, thus, are reasonably justified.

\section{Influence of surface tension}

The influence of surface tension upon the primary breakup was studied with help of two virtual fluids GLW1 and GLW3, which were derived from the experimentally and numerically investigated $G L W 2[23]$ by adjustment of the surface tension to $10 \mathrm{mN} \mathrm{m}^{-1}$ and $100 \mathrm{mNm}^{-1}$, respectively. By this procedure it is possible to isolate the influence of this property. From the Wenumbers given in Tab. 1 one would expect a strong change of the primary breakup morphology from case $G L W 1$ to $G L W 3$, as they differ in one order of magnitude.

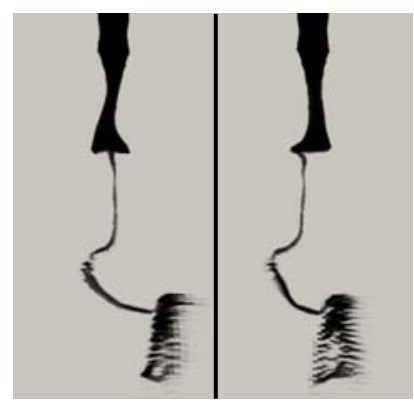

(a) CASE GLW1 $\left(10 \mathrm{mNm}^{-1}\right)$

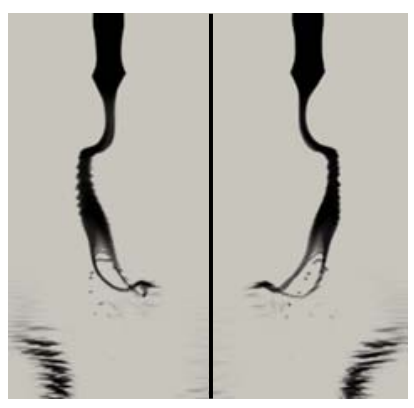

(b) CASE GLW3 $\left(100 \mathrm{mNm}^{-1}\right)$
FIGURE 11: INFLUENCE OF THE SURFACE TENSION UPON THE MORPHOLOGY OF LIQUID JET BREAKUP

Figure 11 shows case GLWI (left) and GLW3 (right) from different view directions. Comparison shows, that the primary breakup morphology stays the same and the breakup regime still is of the membrane type. Influence of the surface tension is only apparent during the formation of the thin ligaments and the membranes, as the decrease of their diameter and therefore the increase of the curvature enhances the impact of surface tension forces.

Additionally, the cases were investigated with regard to breakup length $L_{b}$, frequency $f_{b}$ and the radial expansion of the liquid structures in terms of spray angle $\alpha$, which was analogously determined to [22]. The results are listed in Tab. 3 . Comparing them, a clear trend emerges: An increase of the surface tension leads to an increase of the breakup length and a decrease of the breakup frequency and the radial expansion of the jet, as the stabilizing surface tension forces increase.
TABLE 3: QUANTITATIVE INFLUENCE OF THE SURFACE TENSION UPON THE PRIMARY BREAKUP

\begin{tabular}{lccc|ccc}
\hline & \multicolumn{3}{c}{ SIM } & \multicolumn{3}{c}{ EXP } \\
\cline { 2 - 7 } & $L_{b}$ & $f_{b}$ & $\alpha$ & $L_{b}$ & $f_{b}$ & $\alpha$ \\
Case & $\mathrm{mm}$ & $\mathrm{Hz}$ & $\circ$ & $\mathrm{mm}$ & $\mathrm{Hz}$ & $\circ$ \\
\hline GLW1 & 11.05 & 469 & 21 & - & - & - \\
GLW2 & 11.2 & 428 & 17 & 10.8 & 469 & 27 \\
GLW3 & 11.5 & 410 & 15 & - & - & - \\
\hline
\end{tabular}

* Published in 23 .

\section{Simulation of a suspension with very small particles with a simplified approach}

The influence of non-Newtonian flow behaviour was also investigated for two additional cases $C C G 1$ and $C C G 2$. In this cases, the used fluid represents a suspension with very small particles of approx. $2 \mu \mathrm{m}$ in diameter.

As the VOF-method does not incorporate a description of solid particles in the liquid phase the suspension is modelled by a virtual liquid. This liquid includes the influence of particles by its physical properties, e.g. the density used is equal to that of the suspension (see Tab. 2 for the specific properties). By this approach the numerical effort of the simulations is drastically reduced compared to a modelling of the particles, as neither a Eulerian-Lagrangian method needs to be implemented nor that the particles needs to be discretized by third phase.

The two cases were also evaluated with regard to the apparent viscosity. The breakup length and their comparison with experimental data, as far as available, is shown in Tab. 4. The simulations predict the apparent viscosity within reasonable agreement to the experimental data as well as the general trend. Moreover, the simulations show a decrease of the mean breakup length with an increase of the $G L R$ from case $C C G 1$ to $C C G 2$, which is in agreement with literature, as the momentum ratio increases and most correlations use the following relation: $L_{b} \propto M^{-a}$ [6, 10].

\section{Simulation of CWS with a simplified approach}

In a last step, the breakup of two CWS was studied in cases $C W S 1$ and $C W S 2$, which were motivated by the results of a previous publication [22]. The main goal of this experiment was to investigate the stabilization of CWS due to the formation of capillary suspensions by addition of an immiscible, secondary fluid and its influence upon the atomisation behavoiur. The results of the experiments showed, that the addition of a small amount of octanol drastically improved the sedimentation properties and also improved the atomisation behaviour, as the SMD decreased 
TABLE 4: COMPARISON OF SIMULATION AND EXPERIMENT FOR A NON-NEWTONIAN LIQUID AND A SUSPENSION

\begin{tabular}{lcc|cc}
\hline & \multicolumn{3}{c}{ SIM } & \multicolumn{2}{c}{ EXP } \\
\cline { 2 - 5 } Case & $\begin{array}{c}\mu^{*} \\
\mathrm{mPas}\end{array}$ & $\begin{array}{c}L_{b} \\
\mathrm{~mm}\end{array}$ & $\begin{array}{c}\mu^{*} \\
\mathrm{mPas}\end{array}$ & $\begin{array}{c}L_{b} \\
\mathrm{~mm}\end{array}$ \\
\hline CMC & 156.5 & 10.9 & 152 & 10.8 \\
\hline CCG1 & 161.9 & 9.8 & $185^{*}$ & - \\
CCG2 & 155.5 & 6.6 & 176 & - \\
\hline
\end{tabular}

* Mean value of $176 \mathrm{mPas}(G L R=0.8)$ and $190 \mathrm{mPas}(G L R=0.4)$

with the addition of octanol.

As with the cases $C C G 1$ and $C C G 2$ the same simplified approach was used, which incorporates the particles by global physical properties of the liquid in the VOF-method.

Figure 12 depicts a comparison of the breakup morphology of $C W S 1$ and $C W S 2$ as well as a comparison between experiments and simulations by means of snapshots in a timeseries of $1 \mathrm{~ms}$. In agreement with the experiments, the simulation also predicts the flapping instability with a more pronounced radial deflection of the jet for CWS1 as well as the pulsating instability for CWS2 (The direction of the liquid jet movement is depicted by arrows in Fig. 12. Moreover, the breakup regime is correctly predicted as the fiber type in both cases. In addition, the intact liquid structure, which is still connected with the nozzle exit, has approximately the same size (marked by lines of the same in length in experiment and simulation).

The limitation of the method by the mesh resolution is visible further downstream where the liquid structures disintegrate into smaller droplets in the size of the cells. Nonetheless, the simulations succeed by correctly predicting morphology as well as size and instability mode for both cases with the simplified approach.

\section{CONCLUSION AND OUTLOOK}

Typical non-conventional fuels for EFG feature, amongst other properties, a strong non-Newtonian flow behaviour. This leads to a dependence of the viscosity from the operating conditions of the atomizer in terms of shear stress and therefore complicates the application of typical SMD-correlations (Eqn. (7)).

The influence of the non-Newtonian flow behaviour as well as the influence of the surface tension upon the primary breakup was studied by numerical simulations and validated with experimental data. For this purpose, model equations to describe the shear dependent viscosity of such fuels were implemented in OpenFOAM and successfully validated with a simple test case

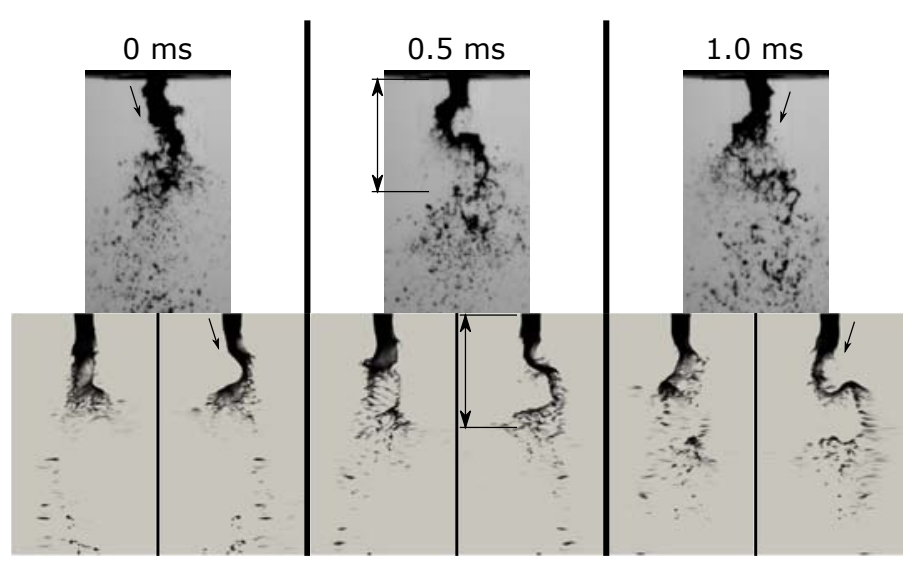

(a) CASE CWS1: TOP: EXPERIMENT. BOTTOM: SIMULATION

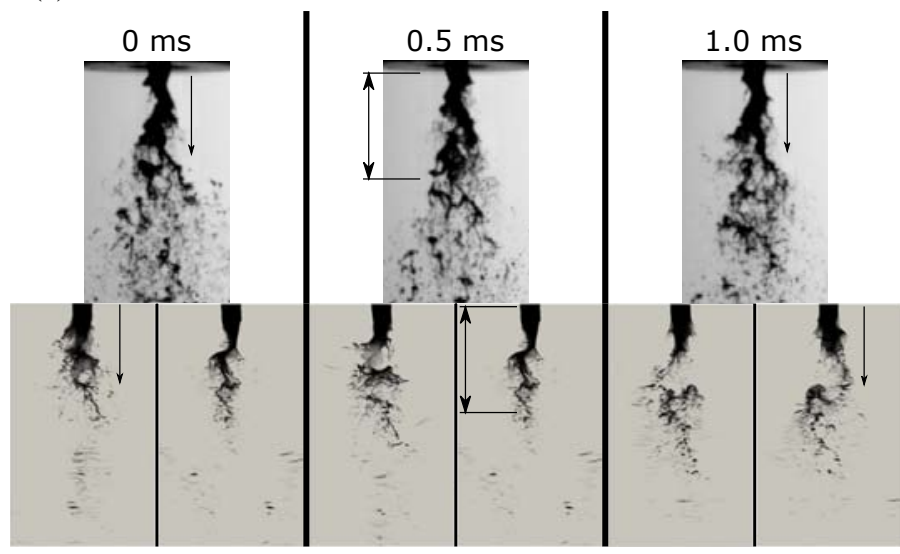

(b) CASE CWS2: TOP: EXPERIMENT. BOTTOM: SIMULATION

FIGURE 12: MORPHOLOGY OF THE PRIMARY BREAKUP

of the Two-plates model. By an extensive comparison of experimental data and the simulation of $C M C$ general applicability of the VOF-method for such liquids was proven. The analysis showed good agreement with regard to the morphology, the primary instability mode as well as quantitative parameters such as the primary breakup length and frequency. Moreover, a dependence of the morphology upon the primary instability mode was confirmed, which is in good agreement with previous studies [25].

Additionally, the influence of surface tension was investigated by simulation of two hypothetical fluids, which differed by one order of magnitude in the surface tension but with otherwise constant properties. The results showed that with increasing surface tension a decrease of the breakup frequency as well as the spray angle due to the increased impact of surface tension forces can be observed. Nevertheless, the quantitative changes of these characteristics are small in comparison to the change of surface tension, which is probably due to the high liquid viscosity.

The influence of shear-thinning flow behaviour by means of 
the apparent viscosity was investigated and compared to experimentally determined values in the region of interest for the primary breakup and showed reasonable agreement with measurements.

In a last step, the primary breakup of two CWS was studied by means of a simplified approach, which used the global properties of the CWS as input parameter for the liquid phase of the VOF-simulations. A comparison of the morphology with regard to characteristic sizes, the breakup regime as well as the primary instability mode showed very good agreement with the experiments. Therefore, the applicability of such simplified models to simulate the primary breakup of such suspension fuels was confirmed.

In order to better understand the atomisation behaviour of high-viscosity fuels in twin-fluid nozzles further experimental and numerical work is ongoing, especially with regard to the influence of the primary instability upon the local stochiometry, nozzle design and scalabilty as well as increased ambient pressure upon the atomisation process.

\section{ACKNOWLEDGMENT}

The authors acknowledge support by the state of BadenWürttemberg through bwHPC. Additionally, the authors like to thank the Helmholtz Association of German Research Centres (HGF) for funding.

\section{REFERENCES}

[1] Higman, C., and van der Burgt, M., 2008. Gasification, 2 ed. Gulf Professional Pub./Elsevier Science, Amsterdam and Boston.

[2] Jakobs, T., Djordjevic, N., Fleck, S., Mancini, M., Weber, R., and Kolb, T., 2012. "Gasification of high viscous slurry R\&D on atomization and numerical simulation". Applied Energy, 93, pp. 449-456.

[3] Dumouchel, C., 2008. "On the experimental investigation on primary atomization of liquid streams". Experiments in Fluids, 45(3), pp. 371-422.

[4] Farago, Z., and Chigier, N., Monday 11 June 1990. "Parametric Experiments on Coaxial Airblast Jet Atomization". In ASME 1990 International Gas Turbine and Aeroengine Congress and Exposition, p. V003T06A016.

[5] Chigier, N., and Farago, Z., 1992. "Morphological Classification of Disintegration of Round Liquid Jets in a Coaxial Air Stream". Atomization and Sprays, 2(2), pp. 137-153.

[6] Lasheras, J. C., Villermaux, E., and Hopfinger, E. J., 1998. "Break-up and atomization of a round water jet by a high-speed annular air jet". Journal of Fluid Mechanics, 357(357), pp. 351-379.

[7] Lasheras, J. C., and Hopfinger, E. J., 2000. "Liquid Jet In- stability and Atomization in a Coaxial Gas Stream". Annual Review of Fluid Mechanics, 32(1), pp. 275-308.

[8] Marmottant, P., and Villermaux, E., 2004. "On spray formation”. Journal of Fluid Mechanics, 498, pp. 73-111.

[9] Eroglu, H., Chigier, N., and Farago, Z., 1991. "Coaxial atomizer liquid intact lengths". Physics of Fluids A: Fluid Dynamics, 3(2), p. 303.

[10] Leroux, B., Delabroy, O., and Lacas, F., 2007. "Experimentel Study of Coaxial Atomizers Scaling. Part I: Dense Core Zone". Atomization and Sprays, 17(5), pp. 381-407.

[11] Leroux, B., Delabroy, O., and Lacas, F., 2007. "Experimentel Study of Coaxial Atomizers Scaling. Part II: Diluted Zone". Atomization and Sprays, 17(5), pp. 409-430.

[12] Tian, X.-S., Zhao, H., Liu, H.-F., Li, W.-F., and Xu, J.-L., 2014. "Effect of central tube thickness on wave frequency of coaxial liquid jet”. Fuel Processing Technology, 119, pp. 190-197.

[13] Jakobs, T., Djordjevic, N., Sanger, A., Zarzalis, N., and Kolb, T., 2015. "Influence of Reactor Pressure on Twinfluid Atomization: Basic Inverstigation on Burner Design for High-Pressure Entrained Flow Gasifier". Atomization and Sprays, 25(12), pp. 1081-1105.

[14] Zhao, H., Liu, H.-F., Xu, J.-L., Li, W.-F., and Cheng, W., 2012. "Breakup and atomization of a round coal water slurry jet by an annular air jet". Chemical Engineering Science, 78(0), pp. 63-74.

[15] Tsai, S. C., and Vu, T., 1987. "Atomization of coalwater slurry using twin-fluid jet atomizer". Fuel, $\mathbf{6 6}(11)$, pp. 1596-1602.

[16] Mulhem, B., Bauckhage, K., Fritsching, U., and Schulte, G., 2003. "Chracterisaton of twin-fluid atomization for suspensions.”. In Institute for Liquid Atomization and Spray Systems, 9th ICLASS Conference.

[17] Sänger, A., Jakobs, T., and Kolb, T., 2016. "Using Primary Instability Analysis for Determination of Apparent Liquid Viscosity at Jet Breakup Atomizing Non-Newtonian Fluids". In Proceedings 27th European Conference on Liquid Atomization \& Spray Systems.

[18] Tian, X.-S., Zhao, H., Liu, H.-F., Li, W.-F., and Xu, J.-L., 2015. "Three-dimensional large eddy simulation of round liquid jet primary breakup in coaxial gas flow using the VOF method". Fuel Processing Technology, 131, pp. 396402.

[19] Gorokhovski, M., and Herrmann, M., 2008. "Modeling Primary Atomization". Annual Review of Fluid Mechanics, 40(1), pp. 343-366.

[20] Sänger, A., Jakobs, T., Djordjevic, N., and Kolb, T., 2014. "Effect of primary instability of a high viscous liquid jet on the spray quality generated by a twin-fluid atomizer". In Proceedings 26th European Conference on Liquid Atomization \& Spray Systems, L. Achelis and U. Fritsching, eds. 
[21] Sänger, A., Jakobs, T., Djordjevic, N., and Kolb, T., 2015. "Experimental investigation on the influence of ambient pressure on twin-fluid atomization of liquids with various viscosities". In Proceedings 13th International Conference on Liquid Atomization \& Spray Systems.

[22] Jampolski, L., Sänger, A., Jakobs, T., Guthausen, G., Kolb, T., and Willenbacher, N., 2016. "Improving the processability of coke water slurries for entrained flow gasification". Fuel, 185, pp. 102-111.

[23] Müller, T., Sänger, A., Habisreuther, P., Jakobs, T., Trimis, D., Kolb, T., and Zarzalis, N., 2016. "Simulation of the primary breakup of a high-viscosity liquid jet by a coaxial annular gas flow". International Journal of Multiphase Flow, 87, pp. 212-228.

[24] Müller, T., Habisreuther, P., Zarzalis, N., Sänger, A., Jakobs, T., and Kolb, T., 2016. "Investigation on Jet Breakup of High-Viscous Fuels for Entrained Flow Gasification”. In ASME Turbo Expo 2016: Turbomachinery Technical Conference and Exposition, p. V003T03A003.

[25] Müller, T., Dullenkopf, A., Habisreuther, P., Zarzalis, N., Sänger, A., Jakobs, T., and Kolb, T., 2017. "Influence of nozzle design upon the primary jet breakup of highviscosity fuels for entrained flow gasification". In ASME Turbo Expo 2017: Turbomachinery Technical Conference and Exposition, p. V003T03A002.

[26] OpenCFD Ltd., 2015. Openfoam - the open source cfd toolbox, http://www.openfoam.org/docs/.

[27] Hirt, C., and Nichols, B., 1981. "Volume of fluid (VOF) method for the dynamics of free boundaries". Journal of Computational Physics, 39(1), pp. 201-225.

[28] Berberovic, E., 2010. "Investigation of Free-surface Flow Associated with Drop Impact: Numerical Simulations and Theoretical Modeling". Ph.d. thesis, Technische Universität, Darmstadt.

[29] Brackbill, J., Kothe, D., and Zemach, C., 1992. "A continuum method for modeling surface tension". Journal of Computational Physics, 100(2), pp. 335-354.

[30] Weller, H. G., 2008. A new approach to VOF-based interface capturing methods for incompressible and compressible flow. Tech. Rep. TR/HGW/04, OpenCFD Ltd.

[31] Zalesak, S. T., 1979. "Fully multidimensional fluxcorrected transport algorithms for fluids". Journal of Computational Physics, 31(3), pp. 335-362.

[32] Leonard, A., 1975. "Energy Cascade in Large-Eddy Simulations of Turbulent Fluid Flows". In Advances in Geophysics: Turbulent Diffusion in Environmental Pollution, Proceedings of a Symposium held at Charlottesville, F. N. Frenkiel and R. E. Munn, eds., Vol. 18, Part A. Elsevier, pp. 237-248.

[33] Smagorinsky, J., 1963. "General circulation experiments with the primitive equations". Monthly Weather Review, 91(3), pp. 99-164.
[34] Fureby, C., 1996. “On subgrid scale modeling in large eddy simulations of compressible fluid flow". Physics of Fluids, 8(5), p. 1301.

[35] Byron Bird, R., and Carreau, P. J., 1968. "A nonlinear viscoelastic model for polymer solutions and melts - I". Chemical Engineering Science, 23(5), pp. 427-434.

[36] Carreau, P. J., 1972. "Rheological equations from molecular network theories". Transactions of the Society of Rheology, 16(1), pp. 99-127.

[37] Cross, M. M., 1965. "Rheology of non-newtonian fluids: A new flow equation for pseudoplastic systems". Journal of Colloid Science, 20(5), pp. 417-437.

[38] Sisko, A. W., 1958. "The flow of lubricating greases". Industrial \& Engineering Chemistry, 50(12), pp. 1789-1792.

[39] Mezger, T., 2014. The rheology handbook: for users of rotational and oscillatory rheometers, 4 ed. European coatings tech files. Vincentz Network, Hannover.

[40] Searle, G. F. G., 1912. "Simple Viscometer for very Viscous Liquids". Proceedings of the Cambridge Philosophical Society, 16, p. 600.

[41] du Noüy, P. L., 1925. "An Interfacial Tensiometer for Universal Use". The Journal of General Physiology, 7, pp. 625-631. 
Karlsruher Institut für Technologie

\section{Repository KITopen}

Dies ist ein Postprint/begutachtetes Manuskript.

\section{Empfohlene Zitierung:}

Müller, T.; Kadel, K.; Habisreuther, P.; Trimis, D.; Zarzalis, N.; Sänger, A.; Jakobs, T.; Kolb, T.

Simulation of the Primary Jet Breakup of Non-Newtonian Fuels: Basic Research for Simulation-Assisted Design of Low-Grade Fuel Burner.

2018. ASME Turbo Expo : Turbomachinery Technical Conference and Exposition GT2018, Oslo, Norway, June 11-15, 2018. Vol.: 3

doi:10.5445/IR/1000085899

Zitierung der Originalveröffentlichung:

Müller, T.; Kadel, K.; Habisreuther, P.; Trimis, D.; Zarzalis, N.; Sänger, A.; Jakobs, T.; Kolb, T.

Simulation of the Primary Jet Breakup of Non-Newtonian Fuels: Basic Research for Simulation-Assisted Design of Low-Grade Fuel Burner.

2018. ASME Turbo Expo : Turbomachinery Technical Conference and Exposition GT2018, Oslo, Norway, June 11-15, 2018. Vol.: 3, Article no: 75945, The American Society of Mechanical Engineers (ASME)

doi:10.1115/GT2018-75945 